\title{
PREVALÊNCIA E PERFIL DE SENSIBILIDADE DE Staphylococcus aureus ISOLADOS EM UM HOSPITAL ESCOLA NA CIDADE DE SANTA MARIA, BRASIL ${ }^{*}$
}

\author{
Maísa Kräulich Tizotti ** \\ Rosmari Horner *** \\ Cláudia Barbisan Kempfer ** \\ Rosiéli Martini ** \\ Letícia Eichstaedt Mayer ** \\ Magda Roehrs ** \\ Mônica de Abreu Rodrigues **** \\ Fábio Teixeira Kuhn **** \\ Adriane Regina Veit $* * * *$ \\ Silvana Oliveira dos Santos ****
}

\section{RESUMO}

Objetivou-se caracterizar a prevalência e o perfil de suscetibilidade de Staphylococcus aureus isolados no Hospital Universitário de Santa Maria (HUSM), através de levantamento de dados junto ao Laboratório de Análises Clínicas, durante o período de abril a junho de 2009. Foram solicitadas 3270 culturas, sendo que 1123 (34\%) resultaram positivas. A prevalência de $S$. aureus em relação às culturas positivas foi de 89 (7,93\%). Verificou-se que $33 \%$ das cepas foram resistentes à oxacilina e que $34,45 \%$ apresentaram resistência relacionada ao gene erm. Elevada sensibilidade ao sulfametoxazol-trimetoprima $(72 / 80 \%)$ foi evidenciada pela automação e nenhuma cepa mostrou-se resistente à vancomicina. Portanto, a prevalência de S. aureus no HUSM e a resistência à oxacilina foram inferiores ao observado em outros hospitais terciários brasileiros.

Descritores: Prevalência; Staphylococcus aureus; Infecção bacteriana; Oxacilina.

\begin{abstract}
The objective of this study was to characterize the prevalence and susceptibility profile of Staphylococcus aureus isolates at University Hospital of Santa Maria, through data collection at the Laboratory of Clinical Analysis, during April to June 2009. Were requested 3270 cultures and 1123 (34\%) were positive. The prevalence of $S$. aureus in relation to positive cultures was 89 (7,93\%). It was found that these strains, 33\% were resistant to oxacillin and $34.45 \%$ showed resistance related erm gene. High sensitivity to trimethoprim-sulfamethoxazole (72/80\%) was detected for the automation and no strain showed resistance to vancomycin. Therefore, the prevalence of $S$. aureus and oxacillin resistance at HUSM were lower than observed in other tertiary hospitals in Brazil.

Descritors: Prevalence; Staphylococcus aureus; Bacterial infection, Oxacillin.
\end{abstract}

\footnotetext{
${ }^{*}$ Trabalho desenvolvido no Departamento de Análises Clínicas e Toxicológicas, Universidade Federal de Santa Maria (UFSM), Santa Maria, RS.

** Curso de Pós-Graduação em Ciências Farmacêuticas, Universidade Federal de Santa Maria, Santa Maria, RS.

*** Departamento de Análises Clínicas e Toxicológicas, Universidade Federal de Santa Maria, Santa Maria, RS.

${ }^{* * * *}$ Curso de Farmácia, Universidade Federal de Santa Maria, Santa Maria, RS.
} 


\section{INTRODUÇÃO}

Staphylococcus aureus tem sido descrito, em diversos estudos nacionais e internacionais como um dos principais agentes etiológicos de infecções humanas ${ }^{1,2}$. Habitualmente, ele constitui a microbiota transitória da pele e de outros sítios anatômicos, sendo o vestíbulo nasal o seu principal reservatório. Esta colonização assintomática representa um fator de risco para infecções endógenas e também tem sido associada à disseminação do microrganismo, principalmente no ambiente hospitalar ${ }^{3}$.

O potencial patogênico do $S$. aureus reside na combinação da virulência mediada por enzimas e toxinas, caráter invasivo e rapidez no desenvolvimento de resistência aos antimicrobianos ${ }^{4}$. Por estes motivos, ele é reconhecido como um patógeno versátil capaz de ocasionar desde processos infecciosos simples até infecções sistêmicas graves, de elevada morbidade e mortalidade ${ }^{4,5}$.

Cepas de $S$. aureus resistentes às penicilinasbetalactamase estáveis, como meticilina e oxacilina, (Methicillin-resistant Staphylococcus aureus MRSA/ORSA) foram relatadas no início da década de 60, logo após a introdução destes antimicrobianos na prática clínica. Esta resistência surgiu devido à integração de um elemento genético móvel, denominado Staphylococcal cassette chromosome mec (SCCmec), em sítio específico do genoma bacteriano $^{6,7,8}$.

O SCCmec contém, além de outros elementos, o gene mecA e seus reguladores (mecl e mecRl), o qual codifica a produção de proteínas ligadoras de penicilina (PBPs) adicionais e alteradas, denominadas PBP2a ou PBP2', que apresentam baixa afinidade por antibióticos betalactâmicos ${ }^{6,7,8}$. Embora haja presença do marcador genotípico (gene $m e c A$ ), a expressão fenotípica da resistência é variável, podendo ser homogênea ou heterogênea ${ }^{7}$.

A prevalência de cepas MRSA associadas aos serviços de saúde, também denominadas HA-MRSA (healthcare-associated MRSA), é variável, dependendo do país, instituição ou setor hospitalar em estudo ${ }^{1,9}$. No Brasil, os índices encontrados são, em média, bastante elevados (40\% a $80 \%)$ especialmente, entre as cepas isoladas em unidades de terapia intensiva $(\mathrm{UTIs})^{10}$. Os fatores de risco relacionados a infecções por HAMRSA incluem geralmente, idade superior a 60 anos, uso de corticóides, uso prévio de antibióticos, internação prolongada e presença de dispositivos médicos invasivos ${ }^{5,11}$.

Nos últimos anos, verificou-se a emergência de infecções comunitárias ocasionadas por MRSA. As cepas responsáveis por essas infecções são conhecidas como CA-MRSA (community-acquired MRSA), e surgiram na comunidade com características clínicas, epidemiológicas e moleculares distintas dos HAMRSA. Normalmente, estão relacionadas a infecções de tecidos e partes moles e apesar de sua maior sensibilidade a antibióticos não betalactâmicos, têm gerado maiores índices de mortalidade $5,12,13,14$.

Os glicopeptídeos, como vancomicina e teicoplanina, ainda constituem a terapêutica de escolha nas infecções por MRSA. Contudo, relatos de cepas de $S$. aureus apresentando suscetibilidade reduzida aos glicopeptídeos em diversos países, incluindo o Brasil, têm levado a dilemas terapêuticos na prática clínica 9 .

Neste estudo, objetivou-se determinar o perfil de sensibilidade aos antimicrobianos e a prevalência de $S$. aureus isolados no Hospital Universitário de Santa Maria (HUSM), durante o período de abril a junho de 2009.

\section{MATERIAIS E MÉTODOS}

Desenvolveu-se um estudo descritivo transversal quantitativo através da análise de laudos de exames culturais realizados no Setor de Bacteriologia do Laboratório de Análises Clínicas do HUSM (LAC HUSM), durante os meses de abril, maio e junho de 2009.

Computou-se o número total de exames culturais solicitados no período, bem como, o número total de culturas positivas. Das culturas nas quais $S$. aureus foi isolado (identificação utilizando automação MicroScan ${ }^{\circledR}$ - Siemens), foram documentados dados referentes ao espécime clínico, caracterização do paciente e teste de sensibilidade aos antimicrobianos (TSA), realizado a partir do painel para gram-positivos (automação) e pelo método convencional de difusão do disco, conforme critérios do Clinical and Laboratory Standards Institute (CLSI 2009) $)^{15}$. Documentou-se também resultados de culturas polimicrobianas, ou 
seja, onde mais de um microrganismo foi isolado, além de $S$. aureus.

\section{ASPECTOOS ÉTICOS}

Este estudo recebeu a aprovação do Comitê de Ética em Pesquisa da Universidade Federal de Santa Maria, sob número 0117.0.243.000-08

\section{RESULTADOS}

Durante o período de abril a junho de 2009, foram solicitados 3270 exames culturais ao LAC-HUSM e deste total, $1123(34,3 \%)$ resultaram positivos. Das culturas positivas, em $65(5,79 \%)$ houve o isolamento de $S$. aureus e em 24 (2,14\%) isolou-se $S$. aureus e outros microrganismos (culturas polimicrobianas), totalizando 89 isolados de $S$. aureus. Dessa forma, a prevalência de $S$. aureus em relação ao total de culturas solicitadas e ao total de culturas positivas foi de 2,72\% ( 89 de 3270) e 7,93\% (89 de 1123), respectivamente.

$\mathrm{Na}$ Tabela 1 está disposta a distribuição por espécime clínico dos 89 S. aureus isolados de pacientes admitidos no HUSM, durante o período de abril a junho de 2009.

Tabela 1 - Distribuição dos S. aureus isolados no HUSM, durante o período de abril a junho de 2009, de acordo com o espécime clínico

\begin{tabular}{cc}
\hline \hline Espécime clínico & $\mathrm{N}(\%)$ \\
\hline Secreções em geral* & $25(28,10)$ \\
Sangue & $19(21,35)$ \\
Aspirado traqueal & $15(16,85)$ \\
Urina & $11(12,36)$ \\
Escarro & $10(11,23)$ \\
Outros $\dagger$ & $9(10,11)$ \\
\hline Total & $89(100)$ \\
\hline \hline
\end{tabular}

* Ferida operatória, escara, lesões, abscesso, coto umbilical, secreção ocular.

$\dagger$ Lavado broncoalveolar, biomateriais (ponta de cateter e de dreno), líquido peritoneal, líquido ascítico.

O Quadro 1 apresenta a listagem dos antimicrobianos sugeridos pelo CLSI 2009 para a realização do TSA, dispostos conforme as metodologias de automação e difusão do disco, sendo classificados de acordo com o grupo do CLSI 2009 ao qual pertencem: Grupo A (primeira escolha, testados e reportados na rotina), Grupo B (primeira escolha, testados e reportados seletivamente, agentes importantes principalmente em infecções nosocomiais), Grupo C (suplementares e reportados seletivamente, agentes alternativos quando ocorrem cepas resistentes a várias drogas primárias), Grupo U (testados em isolados de urina), Grupo O (outros não rotineiramente utilizados) e Grupo Inv. (em investigação) ${ }^{15}$. 
Quadro 1 - Antimicrobianos testados na automação (painel para gram-positivos) e difusão do disco para os S. aureus isolados no HUSM, conforme critérios do CLSI 2009

\begin{tabular}{|c|c|c|c|}
\hline \multicolumn{2}{|c|}{ Automação MicroScan®-Siemens } & \multicolumn{2}{|c|}{ Difusão do disco } \\
\hline Antimicrobiano & Grupo & Antimicrobiano & Grupo \\
\hline Amoxicilina/Ácido & $\mathrm{O}$ & Azitromicina & A \\
\hline Clavulânico & $\mathrm{O}$ & Clindamicina & A \\
\hline Ampicilina/Sulbactam & $\mathrm{O}$ & Teicoplanina & Inv. \\
\hline Ampicilina & $\mathrm{O}$ & Tigeciclina* & - \\
\hline Cefazolina & $\mathrm{O}$ & Oxacilina & A \\
\hline Ceftriaxona & $\mathrm{C}$ & Cefoxitina $\dagger$ & - \\
\hline Ciprofloxacina & A & Sulfametoxazol/Trimetoprima & A \\
\hline Clindamicina & A & & \\
\hline Eritromicina & $\mathrm{C}$ & & \\
\hline Gentamicina & $\mathrm{C}$ & & \\
\hline Levofloxacina & $\mathrm{U}$ & & \\
\hline Nitrofurantoína & $\mathrm{U}$ & & \\
\hline Norfloxacina & A & & \\
\hline Oxacilina & A & & \\
\hline Penicilina & A & & \\
\hline Rifampicina & B & & \\
\hline Sulfametoxazol/Trimetoprima & A & & \\
\hline Vancomicina $\$$ & B & & \\
\hline
\end{tabular}

* O antimicrobiano tigeciclina não consta na listagem do CLSI 2009, mas foi aprovado pelo Food And Drug Administration (FDA) em junho de $2005^{16}$.

† A cefoxitina é agente antimicrobiano para predição de resistência mediada pelo gene mecA em $S$. aureus. O teste com o disco de cefoxitina tem a finalidade de detectar a resistência à oxacilina e não à própria cefoxitina, de maneira que ele é utilizado para reportar sensibilidade ou resistência à oxacilina ${ }^{15}$.

\$ Conforme o CLSI 2009, o teste com o disco de vancomicina não diferencia cepas de S. aureus suscetíveis à vancomicina dos isolados com resistência intermediária, sendo recomendados testes quantitativos para determinar a CIM (Concentração Inibitória Mínima) da vancomicina ${ }^{15}$.

Dentre as 89 culturas positivas para $S$. aureus, duas foram excluídas do estudo dos perfis de sensibilidade antimicrobiana através do método de difusão do disco, devido à insuficiência de dados. Além disso, uma terceira cultura apresentou duas cepas de $S$. aureus com perfis de sensibilidade distintos (heterorresistência), sendo incluídas no estudo dos perfis de sensibilidade antimicrobiana pelo método de difusão do disco e automação. Sendo assim, 88 cepas foram consideradas na caracterização dos perfis de sensibilidade pela difusão do disco (Tabela 2) e 90 na caracterização dos perfis de sensibilidade pelo método automatizado (Tabela 3). 
Tabela 2 - Perfis de sensibilidade antimicrobiana de 88 cepas de $S$. aureus isoladas no HUSM, de acordo com o método de difusão do disco, no período de abril a junho de 2009

\begin{tabular}{|c|c|c|c|c|c|c|c|c|c|}
\hline PERFIL & TGC & TEC & $\mathrm{CFO}$ & $\mathrm{OXA} \dagger$ & SFT $\dagger$ & CLI & AZI & $\mathrm{N}$ & $\%$ \\
\hline \multirow{4}{*}{ I } & $\mathrm{S}$ & $\mathrm{S}$ & $\mathrm{S}$ & - & $\mathrm{S}$ & $\mathrm{S}$ & $\mathrm{S}$ & 13 & \\
\hline & S & S & S & S & - & S & S & 11 & 50,00 \\
\hline & S & S & S & $\mathrm{S}$ & S & S & S & 10 & \\
\hline & $\mathrm{S}$ & $\mathrm{S}$ & $\mathrm{S}$ & - & - & S & S & 10 & \\
\hline \multirow{3}{*}{ II } & S & S & $\mathrm{R}$ & $\mathrm{R}$ & - & $\mathrm{R}$ & $\mathrm{R}$ & 8 & \\
\hline & S & S & $\mathrm{R}$ & - & $\mathrm{R}$ & $\mathrm{R}$ & $\mathrm{R}$ & 4 & 15,87 \\
\hline & S & S & $\mathrm{R}$ & $\mathrm{R}$ & $\mathrm{R}$ & $\mathrm{R}$ & $\mathrm{R}$ & 2 & \\
\hline \multirow{3}{*}{ III } & $\mathrm{S}$ & $\mathrm{S}$ & $\mathrm{S}$ & - & - & $\mathrm{R}$ & $\mathrm{R}$ & 4 & \\
\hline & S & S & S & S & - & $\mathrm{R}$ & $\mathrm{R}$ & 3 & 9,05 \\
\hline & S & S & S & - & S & $\mathrm{R}$ & $\mathrm{R}$ & 2 & \\
\hline \multirow{3}{*}{ IV } & S & S & $\mathrm{R}$ & - & - & $\mathrm{R}$ & $\mathrm{R}$ & 4 & \\
\hline & S & S & $\mathrm{R}$ & - & S & $\mathrm{R}$ & $\mathrm{R}$ & 2 & 7,00 \\
\hline & $\mathrm{S}$ & $\mathrm{S}$ & $\mathrm{S}$ & - & - & $\mathrm{S}$ & $\mathrm{R}$ & 2 & \\
\hline \multirow[t]{2}{*}{$\mathrm{V}$} & S & S & S & S & S & S & $\mathrm{R}$ & 1 & 4,51 \\
\hline & S & S & S & S & - & S & $\mathrm{R}$ & 1 & \\
\hline \multirow{3}{*}{ VI } & $\mathrm{S}$ & $\mathrm{S}$ & $\mathrm{S}$ & - & - & I & I & 1 & \\
\hline & S & S & S & S & - & I & S & 1 & 3,41 \\
\hline & S & S & S & - & - & S & I & 1 & \\
\hline VII & $\mathrm{S}$ & $\mathrm{S}$ & $\mathrm{R}$ & - & - & $\mathrm{S}$ & $\mathrm{S}$ & 3 & 3,41 \\
\hline VIII & $\mathrm{S}$ & $\mathrm{S}$ & $\mathrm{S}$ & - & $\mathrm{R}$ & $\mathrm{R}$ & $\mathrm{R}$ & 2 & 2,23 \\
\hline IX & $\mathrm{S}$ & $\mathrm{S}$ & $\mathrm{S}$ & $\mathrm{S}$ & $\mathrm{R}$ & $\mathrm{S}$ & $\mathrm{R}$ & 2 & 2,23 \\
\hline $\mathrm{X}$ & $\mathrm{S}$ & $\mathrm{S}$ & $\mathrm{R}$ & - & - & $\mathrm{R}$ & $\mathrm{S}$ & 1 & 1,14 \\
\hline Total & & & & & & & & 88 & 100 \\
\hline
\end{tabular}

* $\mathrm{TGC}=$ tigeciclina. $\mathrm{TEC}=$ teicoplanina. $\mathrm{CFO}=$ cefoxitina. $\mathrm{OXA}=$ oxacilina. $\mathrm{SFT}=$ sulfametoxazol/trimetoprima. $\mathrm{CLI}=$ clindamicina. $\mathrm{AZI}=$ azitromicina.

$†$ Para grande parte das cepas não foram empregados os discos de OXA (49 cepas) e SFT (50 cepas) na realização do TSA através do método convencional.

O Teste $\mathrm{D}$, realizado para a detectar a resistência induzível à clindamicina, foi positivo em 7 cepas de $S$. aureus (7 de 88 - 8\%), e destas, 6 apresentaram resistência somente a clindamicina e azitromicina pela difusão do disco (perfil III) (Tabela 2). 
Tabela 3 - Sensibilidade antimicrobiana de 90 cepas de $S$. aureus isoladas no HUSM, conforme resultados do método automatizado MicroScan ${ }^{\circledR}$ - Siemens, no período de abril a junho de 2009

\begin{tabular}{cccc}
\hline Antimicrobianos & Sensível & Intermediário & Resistente \\
& $\mathrm{N}(\%)$ & $\mathrm{N}(\%)$ & $\mathrm{N}(\%)$ \\
\hline Clindamicina & $57(63,33)$ & $2(2,22)$ & $31(34,45)$ \\
Eritromicina & $49(54,45)$ & $2(2,22)$ & $39(43,33)$ \\
Oxacilina & $60(66,67)$ & - & $30(33,33)$ \\
Penicilina & $8(8,89)$ & - & $82(91,11)$ \\
Sulfametoxazol/Trimetoprima & $72(80,00)$ & - & $18(20,00)$ \\
\hline \hline
\end{tabular}

Neste estudo, a sensibilidade à oxacilina foi avaliada através dos resultados da difusão do disco de cefoxitina (Tabela 2) e da CIM de oxacilina no método automatizado (Tabela 3). Não foi possível considerar os resultados da difusão do disco de oxacilina, já que este não foi empregado para todos os isolados.

Houve certa variação entre os resultados do método automatizado e do disco de cefoxitina, visto que, seis cepas apresentaram CIM de oxacilina na faixa de resistência, porém, foram sensíveis à cefoxitina. Conforme recomendações do CLSI 2009, as seis cepas discordantes foram consideradas resistentes à oxacilina. Este critério foi utilizado a fim de evitar erros maiores, pois apesar de menos frequentes, existem outros mecanismos de resistência à oxacilina não associados à presença do gene mecA, que envolvem a hiperprodução de $\beta$-lactamases tipo A e modificações nas PBPs habituais ${ }^{8,10}$.

Dessa forma, a prevalência de cepas de $S$. aureus resistentes à oxacilina no HUSM, durante o período de abril a junho de 2009, foi 30 (30 de 90 - 33,33\%). Em relação ao total de culturas solicitadas sua prevalência foi de $0,92 \%$ ( 30 de $3270-0,92 \%$ ).

A Tabela 4 traz a distribuição, por unidade hospitalar, dos $30 \mathrm{~S}$. aureus resistentes à oxacilina isolados no HUSM, durante o período de abril a junho de 2009.

Tabela 4 - Distribuição dos S. aureus resistentes à oxacilina, isolados de pacientes atendidos no HUSM, no período de abril a junho de 2009

\begin{tabular}{lc}
\hline Unidade & $\mathrm{N}(\%)$ \\
\hline Clínica cirúrgica & $6(20,00)$ \\
Clínica ginecológica & $5(16,67)$ \\
Ambulatório & $4(13,33)$ \\
Unidade de terapia intensiva pediátrica & $3(10,00)$ \\
Centro de tratamento de crianças com câncer & $3(10,00)$ \\
Clínica médica & $3(10,00)$ \\
Unidade de terapia intensiva adulta & $2(6,67)$ \\
Pronto atendimento adulto & $2(6,67)$ \\
Pronto atendimento pediátrico & $1(3,33)$ \\
Nefrologia & $1(3,33)$ \\
\hline Total & $30(100)$ \\
\hline
\end{tabular}

Nas Tabelas 5 e 6 estão dispostos os perfis de sensibilidade antimicrobiana das cepas de $S$. aureus resistentes à oxacilina, conforme resultados da metodologia automatizada. 
Tabela 5 - Sensibilidade aos antimicrobianos dos 30 S. aureus resistentes à oxacilina isolados no HUSM, no período de abril a junho de 2009, de acordo com a metodologia automatizada (Microscan $®$ - Siemens)

\begin{tabular}{cccc}
\hline Antimicrobianos & Sensível & Intermediário & Resistente \\
\hline Grupo - CLSI 2009) & $\mathrm{N}(\%)$ & $\mathrm{N}(\%)$ & $\mathrm{N}(\%)$ \\
Penicilina (A) & - & - & $30(100)$ \\
Cefazolina (O) & - & - & $30(100)$ \\
Eritromicina (A) & $4(13,3)$ & - & $26(86,7)$ \\
Clindamicina (A) & $6(20,0)$ & - & $24(80,0)$ \\
Gentamicina (C) & $7(23,3)$ & $1(3,3)$ & $22(73,4)$ \\
Ciprofloxacina (C) & $10(33,3)$ & $2(6,7)$ & $18(60,0)$ \\
Sulfametoxazol/Trimetoprima (A) & $14(46,7)$ & - & $16(53,3)$ \\
Levofloxacina (C) & $11(36,7)$ & $6(20,0)$ & $4(13,3,3)$ \\
Rifampicina (B) & $23(76,7)$ & $3(10,0)$ & - \\
Vancomicina (B) & $30(100)$ & - &
\end{tabular}

Tabela 6 - Perfis de sensibilidade antimicrobiana de $30 \mathrm{~S}$. aureus resistentes à oxacilina isolados no HUSM, no período de abril a junho de 2009, conforme metodologia automatizada (Microscan ${ }^{\circledR}$ - Siemens)

\begin{tabular}{|c|c|c|c|c|c|c|c|c|c|}
\hline PERFIL & VA & RIF & LVX & SFT & $\mathrm{CP}$ & GM & CLI & EI & $\mathrm{N}(\%)$ \\
\hline I & S & $\mathrm{S}$ & $\mathrm{R}$ & $\mathrm{R}$ & $\mathrm{R}$ & $\mathrm{R}$ & $\mathrm{R}$ & $\mathrm{R}$ & $6(20,00)$ \\
\hline II & $\mathrm{S}$ & $\mathrm{S}$ & I & $\mathrm{R}$ & $\mathrm{R}$ & $\mathrm{R}$ & $\mathrm{R}$ & $\mathrm{R}$ & $4(13,33)$ \\
\hline III & $\mathrm{S}$ & $\mathrm{R}$ & $\mathrm{R}$ & $\mathrm{R}$ & $\mathrm{R}$ & $\mathrm{R}$ & $\mathrm{R}$ & $\mathrm{R}$ & $3(10,00)$ \\
\hline IV & $\mathrm{S}$ & $\mathrm{S}$ & $\mathrm{R}$ & $\mathrm{S}$ & $\mathrm{R}$ & $\mathrm{R}$ & $\mathrm{R}$ & $\mathrm{R}$ & $3(10,00)$ \\
\hline V & $\mathrm{S}$ & $\mathrm{S}$ & $\mathrm{S}$ & $\mathrm{S}$ & $\mathrm{S}$ & $\mathrm{S}$ & $\mathrm{S}$ & $\mathrm{S}$ & $3(10,00)$ \\
\hline VI & $\mathrm{S}$ & $\mathrm{S}$ & $\mathrm{S}$ & $\mathrm{S}$ & $\mathrm{S}$ & $\mathrm{S}$ & $\mathrm{R}$ & $\mathrm{R}$ & $2(6,67)$ \\
\hline VII & $\mathrm{S}$ & $\mathrm{R}$ & $\mathrm{R}$ & $\mathrm{R}$ & $\mathrm{S}$ & $\mathrm{R}$ & $\mathrm{R}$ & $\mathrm{R}$ & $1(3,33)$ \\
\hline VIII & $\mathrm{S}$ & $\mathrm{S}$ & I & $\mathrm{R}$ & $\mathrm{R}$ & $\mathrm{R}$ & $\mathrm{S}$ & $\mathrm{R}$ & $1(3,33)$ \\
\hline IX & $\mathrm{S}$ & $\mathrm{S}$ & I & $\mathrm{S}$ & $\mathrm{R}$ & $\mathrm{R}$ & $\mathrm{R}$ & $\mathrm{R}$ & $1(3,33)$ \\
\hline $\mathrm{X}$ & $\mathrm{S}$ & I & $\mathrm{S}$ & $\mathrm{S}$ & $\mathrm{S}$ & $\mathrm{R}$ & $\mathrm{R}$ & $\mathrm{R}$ & $1(3,33)$ \\
\hline XI & $\mathrm{S}$ & I & $\mathrm{S}$ & $\mathrm{S}$ & $\mathrm{S}$ & I & $\mathrm{R}$ & $\mathrm{S}$ & $1(3,33)$ \\
\hline XII & $\mathrm{S}$ & I & $\mathrm{S}$ & $\mathrm{S}$ & I & $\mathrm{R}$ & $\mathrm{R}$ & $\mathrm{R}$ & $1(3,33)$ \\
\hline XIII & $\mathrm{S}$ & $\mathrm{S}$ & $\mathrm{S}$ & $\mathrm{S}$ & $\mathrm{S}$ & $\mathrm{R}$ & $\mathrm{R}$ & $\mathrm{R}$ & $1(3,33)$ \\
\hline IXV & $\mathrm{S}$ & $\mathrm{S}$ & $\mathrm{S}$ & $\mathrm{R}$ & I & $\mathrm{S}$ & $\mathrm{S}$ & $\mathrm{R}$ & $1(3,33)$ \\
\hline XV & $\mathrm{S}$ & $\mathrm{S}$ & $\mathrm{S}$ & $\mathrm{S}$ & $\mathrm{S}$ & $\mathrm{S}$ & $\mathrm{S}$ & $\mathrm{R}$ & $1(3,33)$ \\
\hline Total & & & & & & & & & $30(100)$ \\
\hline
\end{tabular}

* VA = vancomicina, $\mathrm{RIF}=$ rifampicina, $\mathrm{LVX}=$ levofloxacina, $\mathrm{SFT}=$ sulfametoxazol/trimetoprima, $\mathrm{CP}=$ ciprofloxacina, $\mathrm{GM}=$ gentamicina, $\mathrm{CLI}=$ clindamicina, $\mathrm{EI}=$ eritromicina.

\section{DISCUSSÃO}

Dados do Programa de Vigilância Epidemiológica e de Resistência Antimicrobiana (SENTRY 1997 a 2001), que abrangeu hospitais brasileiros e latino- americanos, apontam o $S$. aureus como o patógeno prevalente nas infecções em geral, sendo o primeiro em frequência nas infecções de tecidos e partes moles e bacteremias e o segundo em infecções do trato respiratório inferior ${ }^{17,18}$. Nos Estados Unidos, dados do 
National Nosocomial Infection Surveillance (NNIS), conduzido pelo Centers for Disease Control and Prevention (CDC) apontam o S. aureus como uma das causas mais comuns de infecções nosocomiais, incluindo pneumonias associadas à ventilação mecânica, infecções de sítio cirúrgico e infecções da corrente sangüínea relacionadas a cateter ${ }^{19}$.

Comparando os padrões de prevalência nacional e internacional aos resultados obtidos neste estudo podese dizer que a prevalência do $S$. aureus no HUSM mostrou-se pouco significativa ( 89 de 1123 - 7,93\%), sugerindo que este, provavelmente, não seja o principal agente etiológico na instituição. Nesse sentido, é válida a idéia de que existem diferenças importantes na prevalência de patógenos entre países e também entre hospitais num mesmo país.

Em relação à distribuição do $S$. aureus por espécime clínico (Tabela 1), nota-se similaridade ao relacioná-la aos principais sítios de infecção encontrados na literatura ${ }^{17,18,19}$. No HUSM, o $S$. aureus foi frequente nas secreções em geral (relacionado a infecções de pele ou de sítio cirúrgico), seguido de sangue (bacteremias) e aspirado traqueal (infecção do trato respiratório inferior). Contudo, em amostras de urina sua frequência também foi significativa, demonstrando que nesta instituição o agente pode ter relevância nas infecções do trato urinário.

A partir do exposto na Tabela 2, nota-se que 44 (50\%) isolados de $S$. aureus apresentaram elevada sensibilidade antimicrobiana (perfil I). Em contraste, 14 $(15,87 \%)$ isolados mostraram-se suscetíveis apenas a teicoplanina e a tigeciclina (perfil II). Nos perfis III e IV pode-se evidenciar, presuntivamente, a resistência mediada pelo gene erm, ou seja, resistência à azitromicia e clindamicina ou D-teste positivo $(15 / 16,05 \%)$, sendo que no perfil IV também há resistência mediada pelo gene $m e c A$, caracterizada pela resistência à cefoxitina (6/7\%). $\mathrm{O}$ perfil $\mathrm{V}$ caracterizouse pela resistência mediada pelo gene $m s r A$, ou seja, resistência apenas à azitromicina (4/4,51\%), sendo os perfis subsequentes menos prevalentes (perfil VI a X).

Na Tabela 3 pode-se observar o alto índice de resistência à penicilina entre os isolados (82 de 90 91,11\%), o que está de acordo com dados da literatura nacional ${ }^{9,10}$. Por outro lado, torna-se notável o percentual elevado de sensibilidade ao sulfametoxazol/trimetoprima (72 de $90-80 \%$ ). Os genes erm e msrA foram responsáveis por 43,33\% (39 de 90 - 43,33\%) de resistência aos macrolídeos, corroborando com dados do SENTRY ${ }^{18}$. A resistência mediada pelo gene erm foi significativa (31 de 90 $34,45 \%$ ), apresentando-se constitutiva em 24 isolados (24 de 90 - 26,45\%) e induzível em 7 dos isolados (7 de 90 - 8\%), sendo esta última evidenciada, especialmente, entre as cepas sensíveis à oxacilina. Dados similares foram relatados em estudo internacional, no qual houve $32,1 \%$ de resistência à clindamicina e a resistência induzível esteve presente em 7,8\% dos isolados de $S$. aureus, sendo a maioria destes sensível à oxacilina ${ }^{20}$.

A taxa média de resistência à oxacilina em $S$. aureus, entre os hospitais brasileiros participantes do programa SENTRY, foi de $34 \%{ }^{17}$. Analisando dados de estudos posteriores, em alguns hospitais do país, o índice foi de, aproximadamente, $70 \%{ }^{21,22}$. Dessa forma, pode-se dizer que o percentual encontrado no HUSM (30 de 90 - 33,33\%) é similar ao índice médio descrito no SENTRY, porém, bem menor do que o encontrado em outros hospitais brasileiros. Cabe ressaltar, que a prevalência de cepas MRSA é muito diversificada e depende das características de cada região, dos setores hospitalares, do sítio de infecção e origem (nosocomial ou comunitária) ${ }^{14}$.

Observando a Tabela 4, verifica-se que não há diferenças significativas na prevalência das cepas MRSA entre as unidades hospitalares, mas nota-se uma maior frequência nas unidades cirúrgicas (6/20\%). Diversos fatores têm sido relacionados à incidência de infecções no sítio cirúrgico como aqueles inerentes ao agente infeccioso (tamanho do inóculo) e ao paciente (idade, doenças de base, desnutrição, imunidade), ressaltando a importância do controle das infecções na clínica cirúrgica ${ }^{23}$.

Analisando a Tabela 5, nota-se que as cepas MRSA identificadas neste estudo apresentaram um índice considerável de resistência a diferentes antimicrobianos. Houve resistência acima de 50\% para os antimicrobianos eritromicina, clindamicina, gentamicina, ciprofloxacina e sulfametoxazol/ trimetoprima. Para levofloxacina houve $43,3 \%$ de resistência plena e $20 \%$ de resistência intermediária, sendo um dado preocupante, já que este antimicrobiano 
tem relevância no tratamento de infecções do trato respiratório. A maioria das cepas mostrou-se sensível à rifampicina $(23 / 76,7 \%)$ e todas foram sensíveis à vancomicina, condizendo com a literatura ${ }^{24}$. Todavia, apesar da sensibilidade significativa à rifampicina, o CLSI 2009 não recomenda o uso deste antimicrobiano como tratamento isolado nas infecções ${ }^{15}$.

As cepas com perfis I, II, III, IV, VII mostraram-se resistentes a cinco ou mais antibióticos não betalactâmicos e as cepas com perfis VIII e IX apresentaram resistência a pelo menos quatro antimicrobianos não betalactâmicos (Tabela 6). É possível sugerir que cepas com índice elevado de resistência são características do Clone Endêmico Brasileiro de MRSA (SCCmec tipo IIIA), o qual possui alta probabilidade de aquisição nosocomial ${ }^{24,25}$. Já as cepas com perfil V e XV são atípicas e, provavelmente, albergam o SCCmec tipo IV devido a sua elevada sensibilidade aos antimicrobianos não betalactâmicos, especialmente sulfametoxazol/ trimetoprima e clindamicina. Geralmente as cepas com SCCmec IV estão relacionadas a infecções comunitárias ${ }^{12,13}$, porém, metodologia molecular seria necessária para esta análise.

\section{CONCLUSÕES}

Através dos resultados obtidos neste estudo, conclui-se que a prevalência de $S$. aureus no HUSM mostra-se pouco significativa, ou seja, é menor do que a encontrada em outros nosocômios no Brasil e no exterior.

Verifica-se que a maioria dos isolados apresenta sensibilidade a um número amplo de antimicrobianos e que a presença de cepas resistentes à oxacilina não é tão frequente como em outros hospitais terciários. No entanto, os perfis de sensibilidade das cepas MRSA são preocupantes, já que houve um número considerável de cepas sensíveis somente à vancomicina, teicoplanina e tigeciclina.

O elevado índice de resistência mediada pelo gene erm, principalmente em cepas sensíveis à oxacilina, ressalta a importância da realização do TSA e testes de caracterização de resistência induzível à clindamicina, a fim de se evitar possíveis falhas terapêuticas na prática clínica.
O sulfametoxazol/trimetoprima, devido à alta sensibilidade encontrada neste estudo, pode representar uma opção terapêutica importante para o tratamento de infecções não complicadas em tecidos e partes moles.

A vancomicina pode ser utilizada como tratamento padrão para cepas MRSA neste nosocômio. Todavia, devido à emergência de cepas com suscetibilidade reduzida em diferentes países, sugere-se que seu uso seja efetuado criteriosamente com o objetivo de resguardá-la, prevenindo o surgimento dos VRSA. Outras opções terapêuticas como tigeciclina, daptomicina, quinupristina/dalfopristina, linezolida, ceftobiprole e ceftaroline podem ser utilizadas como alternativa à vancomicina, apresentando eficácia clínica semelhante.

Sendo assim, pode-se dizer que o HUSM apresenta características favoráveis em relação aos índices de infecção por $S$. aureus e MRSA. No entanto, recomenda-se que estudos como este continuem a serem efetuados, a fim de monitorar os programas de controle de infecção e os índices de resistência no cenário local.

\section{REFERÊNCIAS}

1- Oliveira DC; Tomasz A; Lencastre H. Secrets of success of a human pathogen: molecular evolution of pandemic clones of meticillin-resistant Staphylococcus aureus. Lancet 2002; 2:180-189.

2- Trindade PA; Pacheco RL; Costa SF et al. Prevalence of SCCmec type IV in nosocomial bloodstream isolates of methicillin-resistant Staphylococcus aureus. J Clin Microbiol 2005; 43(7):3435-3437.

3- Cavalcanti SMM; França ER; Cabral C et al. Prevalence of Staphylococcus aureus Introduced into Intensive Care Units of a University Hospital. Braz J Infect Dis 2005; 9(1):56-63.

4- Santos AL; Santos DO; Freitas CC et al. Staphylococcus aureus: visitando uma cepa de importância hospitalar. J Bras Patol Med Lab 2007; 43(6):413-423.

5- Lopes HV. CA-MRSA: um novo problema para o infectologista. Rev Panam Infectol 2005; 7(3):34-36.

6- Hiramatsu K, Katayama Y, Yuzawa H. Molecular genetics of methicillin-resistant Staphylococcus aureus. J Med Microbio 2002; 292:67-74. 
7- Deresinski S. Methicillin-resistant Staphylococcus aureus: an evolutionary, epidemiologic, and therapeutic odyssey. Clin Infect Dis 2005; 40:562-573.

8- Chambers HF. Methicillin resistance in staphylococci: molecular and biochemical basis and clinical implications. Clin Microbiol Rev 1997; 10 (4):781-791.

9- Mimica MJ; Mendes CMF. Diagnóstico laboratorial da resistência à oxacilina em Staphylococcus aureus. J Bras Patol Med Lab 2007; 43 (6):399-406.

10- Rossi F; Andreazzi DB. Interpretando o antibiograma. Cocos Gram-positivos: Staphylococcus aureus, Enterococcus spp. e Streptococcus pneumoniae e os principais mecanismos de resistência. São Paulo: Atheneu; 2005, p. 27-63.

11- Ribeiro J; Boyce JM; Zancanaro PQ. Prevalence of methicillin-resistant Staphylococcus aureus (MRSA) among patients visiting the emergency room at a tertiary hospital in Brazil. Braz J Infect Dis 2005; 9(1):52-55.

12- Weber JT. Community-associated methicillinresistant Staphylococcus aureus. Clin Infect Dis 2005; 41:269-272.

13- Ricardo SB. MRSA comunitário: uma nova ameaça. Prática Hospitalar 2006; 46:170-172.

14- Ricardo SB. Emergência da $S$. aureus meticilinaresistente (MRSA) na comunidade. Prática Hospitalar 2004; 34:170-172.

15- Clinical and Laboratory Standards Institute. Performance Standards for Antimicrobial Susceptibility Testing; Nineteenth Informational Supplement Approved Standard M100-S19. CLSI, Wayne, Pensylvania, USA, 2009.

16- Tygacil ${ }^{\mathrm{TM}} \quad$ [tigecycline] package insert. Wyeth Pharmaceuticals Inc, Philadelphia, PA, USA, June 2005.

17- Sader HS; Gales AC; Pfaller MA et al. Pathogen frequency and resistence patters in Brazilian hospitals: summary of results from three years of the SENTRY antimicrobial surveillance program. Braz J Infect Dis 2001; 5(4):200-214.

18- Sader HS; Jones RN; Gales AC et al. SENTRY Antimicrobial Surveillance Program Report: Latin American and Brazilian results for 1997 through 2001. Braz $\mathrm{J}$ Infect Dis 2004; 8(1):25-79.
19- Kuehnert MJ; Hill HA; Kupronis BA et al. Methicillinresistant-Staphylococcus aureus Hospitalizations, United States. Emerg Infect Dis 2005; 11(6):868-872.

20- Delialioglu N; Aslan G; Ozturk C et al. Inducible clindamycin resistance in Staphylococci isolated from clinical samples. Jpn J Infect Dis 2005; 58:104-106.

21- Menezes EA; Sá KM; Cunha FA et al. Freqüência e percentual de suscetibilidade de bactérias isoladas em pacientes atendidos na Unidade de Terapia Intensiva do Hospital Geral de Fortaleza. J Bras Patol Med Lab 2007; 43(3):149-155.

22- Leão LSNO; Passos XS; Reis C et al. Fenotipagem de bactérias isoladas de hemoculturas de pacientes críticos. Rev Soc Bras Med Trop 2007; 40:537-540.

23- Rabhae GN, Ribeiro-Filho N, Fernandes AT. Infecção do sítio cirúrgico. In: Fernandes AT, Fernandes MOV, Ribeiro-Filho, N. Infecção hospitalar e suas interfaces na área da saúde. São Paulo: Atheneu; 2000, p. 479-505.

24- Oliveira GA; Faria JB; Levy CE et al. Characterization of the Brazilian endemic clone of methicillin-resistant Staphylococcus aureus (MRSA) from hospitals throughout Brazil. Braz J Infect Dis 2001; 5(4):163-170.

25- Vivoni AM; Diep BA; Magalhães ACG et al. Clonal composition of Staphylococcus aureus isolates at a Brazilian University Hospital: identification of international circulating lineages. J Clin Microbiol 2006; 44(5):1686-1691.

\section{Endereço para correspondência:}

Prof. Dr ${ }^{\mathrm{a}}$. Rosmari Hörner.

Departamento de Análises Clínicas e Toxicológicas Universidade Federal de Santa Maria

Prédio 26, Sala 1201, Campus UFSM, CEP: 97105-900, Santa Maria, RS.

Recebido em 08 de outubro de 2010.

Aprovado em 18 de novembro de 2010. 